\title{
SINGLE CHIP MICRO GC WITH INTEGRATED HETEROGENEOUS NANOMATERIAL SENSOR ARRAY FOR MULTIPARAMETER GAS SENSING
}

\author{
S. MacNaughton ${ }^{1}$, and S. Sonkusale ${ }^{{ }^{*}}$ \\ ${ }^{1}$ Tufts University, Department of Electrical and Computer Engineering, Medford, MA 02155
}

\begin{abstract}
We present a PDMS micromolded gas chromatography system on chip with integrated gas sample injection, heating elements, as well as a nanomaterial based chemiresistor array for gas detection. This device is the first realization of a micromolded (as opposed to etched) microscale gas chromatography ( $\mu \mathrm{GC}$ ) system. The PDMS mold serves the dual purpose of creating the column geometry as well as acting as the stationary phase during the gas chromatography analysis. A glass wafer acts not only to seal to column but also as a platform for a micropatterned detector and heating element. A chemiresitor array consisting of carbon nanotubes $(\mathrm{CNT})$, vanadium oxide nanowires $\left(\mathrm{VO}_{\mathrm{x}}\right)$ and reduced graphene oxide (rGO) provide cross-calibrated detection of gas constituents after chromatographic separation to yield a multiparameter gas analysis.
\end{abstract}

\section{INTRODUCTION}

Conventional gas sensors excel at identifying single gas analyte but are confounded by mixtures of gases. Therefore, gas chromatography is a pillar of gas analysis due to its ability to separate the constituents of a gas phase mixture. However, current available gas chromatography systems are bulky benchtop devices, which necessitate sampling gases and returning the samples for analysis. Because this process consumes time and has the potential to compromise gas analysis, there is a need for portable, lowpower gas chromatography devices for use in the field. Much research has been dedicated to the realization of highly effective, miniaturized gas chromatography systems over the past three decades[1, 2]. However, despite their successes, no commercial development of micro-gas chromatography $(\mu \mathrm{GC})$ devices has been realized. The reasons for this is two-fold: first, the majority of $\mu \mathrm{GC}$ systems developed do not incorporate micro-scale components for all facets of the measurement, so they still rely on bulky components, which undermine their utility versus a conventional benchtop device. Second, the typical fabrication process for these devices (reactive ion etching, anodic bonding and stationary phase coating) is complex, difficult to reproduce, and requires expensive vacuum and plasma systems. The presented device includes all the necessary components for gas chromatography in a low cost, easily fabricated device.

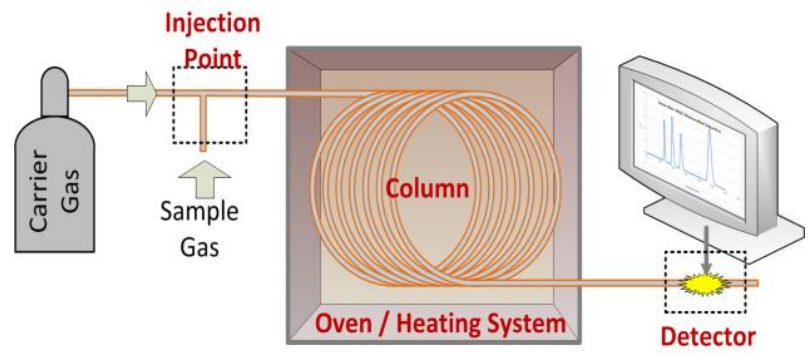

Figure 1 - Schematic representation of conventional gas chromatography device

Gas chromatography separates the constituents of a sample of gas mixture based upon their interactions with the sidewall of a long tube known as the gas column. The column is coated with a special adsorbent material known as the stationary phase which is often a waxy polymer or silicone. As the gas sample is pushed through the column by a carrier gas, analytes with stronger affinity for the stationary phase adsorb and condense making them travel though the column more slowly. A detector at the end of the column registers when each gas species elutes after a specific characteristic time known as the retention time. The miniaturization of columns aids in the separation efficiency of chromatography columns as it increases the surface area per volume of gas analyte.

The general components of a gas chromatography system are shown in Figure 1. These components are the injection system, the heating element, the detector, and, finally, the detector itself. Each of these components presents distinct challenges in their miniaturization and will be detailed in the ensuing sections.

\section{Injection Mechanism}

Micromachined gas columns place especially onerous constraints on the gas sample injection strategy due to the especially small volumes of gases that are injected. Generally, volumes on the order of nanoliters or less are required. An effective and common strategy to achieve adequate injection is illustrated in Figure 2. A cross-flow pattern is used to capture a small pocket of the gas sample and direct it through the column [3]. This process is mediated by high-speed valves (Clark Solutions, Model number 2026).

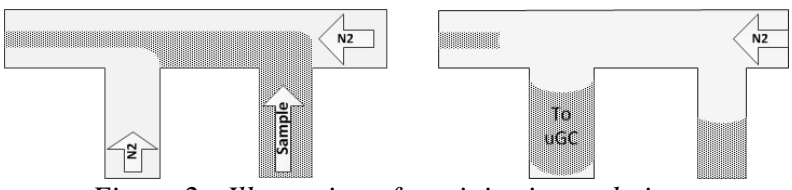

Figure 2 - Illustration of gas injection technique.

\section{Detector}

A chemiresistor array was chosen as the detection strategy. Chemiresistors are natural microscale allowing for their facile integration into a microscale column. Furthermore, an array allows for multiparameter analysis of gas constituents based upon the magnitude of the response to the various elements in the heterogeneous array. Nanoscale chemiresistors also possess the necessary response time for time domain analyses as in gas chromatography. Carbon (reduced graphene oxide and carbon nanotubes) and metal oxide nanowires (vanadium oxide) were chosen as chemiresistive elements due to prior experience with the materials [4].

Drawbacks of chemiresistor array include a memory effect and lack of correlation between analyte quantity and magnitude of response. Chemiresistors are known to have their response be a function of not only the current chemical environment, but also their chemical history. Methods have been developed to account for this [5]. The lack of correlation between response magnitude and analyte quantity makes it difficult to determine the relative concentrations of each constituent of the gas mixture. Although all 

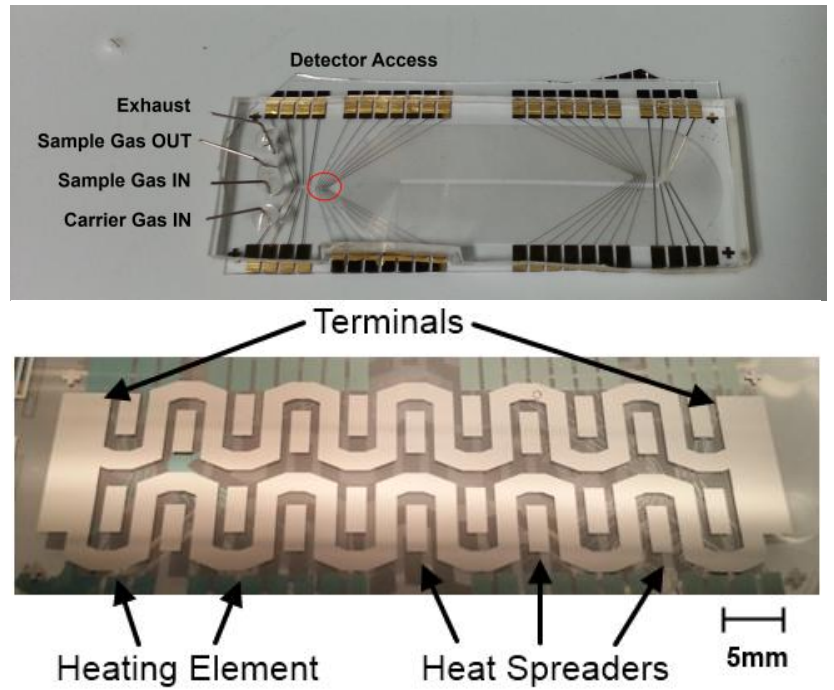

Figure 3 - Image of fabricated device from above (top) and below (bottom). The heating element is removed from the top picture to make the column spiral more visible versus the white background. The device is same size as a common microscope slide $(25 \mathrm{~mm} x$ $75 \mathrm{~mm}$ ). The chemiresistor detector is circled in red.

detectors have to be calibrated by weighting coefficients for any individual analyte, this is much more pronounced in the case of chemiresistors. However, it is this dynamic range of response which allows another parameter upon which to identify gas components.

\section{Heating Element}

The interaction between the stationary phase and gas samples is very dependent on temperature. As such it is critical to be able to maintain an accurate and consistent temperature profile throughout the course of the measurement. A nichrome heating element (200nm thick) patterned directly on the device accomplishes this (see Figure 3).

The heating element drives the power consumption of the device, which is of prime importance when designing for use in the field under battery power. The heating element is able to maintain the temperature of the device at $100^{\circ} \mathrm{C}$ with 1.67 watts of power, very much within the capability of batteries. The temperature is monitored with a thermocouple.

\section{Column}

Finally, the most important element of the design is the column. Polydimethylsiloxane (PDMS) and similar compounds have long been used as excellent stationary phases for the separation of nonpolar analytes in gas chromatography, and, with the massive volume of interest in PDMS for microfluidics, it would seem that gas chromatography would be a natural application for micromolded PDMS. However, the high permeability of PDMS to many gas compounds presents a significant hurdle in the development of a micromolded gas column. With micromolded columns, there are two parallel paths through the column. The first (preferred) path is through the column as in a conventional capillary column. The second parasitic path is driven by the permeation of gas analytes through the walls of the column between adjacent turns.

There are two strategies to mitigate this in designing the column geometry. First, the column walls can be made very thick. This presents a high resistance to permeation, but limits the amount of turns and therefore column length which can drive down separation efficiency. The second strategy is to increase the cross sectional area of the column, which conversely reduces the resistance of flow propagating through the column, but also limits the interaction between the gas sample and column sidewalls.

A combination of these two strategies was used. An increased wall thickness was used in the first third of the column where the pressure differential and therefore permeation between adjacent turns is greatest. A modest column width and height of nominally $20 \mu \mathrm{m}$ was used for the entirety of the column, which was $10 \mathrm{~m}$ in length. The length of the column was chosen to be rather long as a conservative measure in case the separation efficiency was lower than expected so that successive peaks could still be resolved.

\section{METHODS}

\section{Fabrication}

The fabrication of the device is represented in Figure 4. The fabrication proceeds as follows. A PDMS micromold containing the column geometry is created using standard micromolding techniques with SU-8 photolithography. Concurrently, a glass wafer is patterned on two sides by standard photolightography, metallization (sputtering) and liftoff. The first side is patterned with gold electrodes and pads for the chemiresistor array and its monitoring. The obverse side is patterned with the heating element pattern shown in Figure 3.

Dielectrophoresis is used to assemble nanomaterials (reduced graphene oxide flakes, carbon nanotubes and vanadium oxide nanowires). Dielectrophoresis is the motion of a polarizable particle in a dielectric medium as a result of a time-varying electric field, and it can be used to pull nanomaterials out a liquid dispersion to form a resistive assembly between two electrodes. This process is described in detail in previous work [6].

The nanomaterials were procured as follows: the reduced graphene oxide was synthesized in the procedure described in previous work. The carbon nanotubes were procured from Helix Material Solutions, and the $\mathrm{VO}_{\mathrm{x}}$ nanowires from Novarials Corporation.

Once the chermiresistor array is created the glass wafer is subjected to a low power (50W) oxygen plasma for 30s at 200Torr. This allows covalent bonding between the PDMS micromold and glass wafer, thus sealing the column.
Glass Sealing Wafer

1.

2.

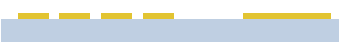

3.

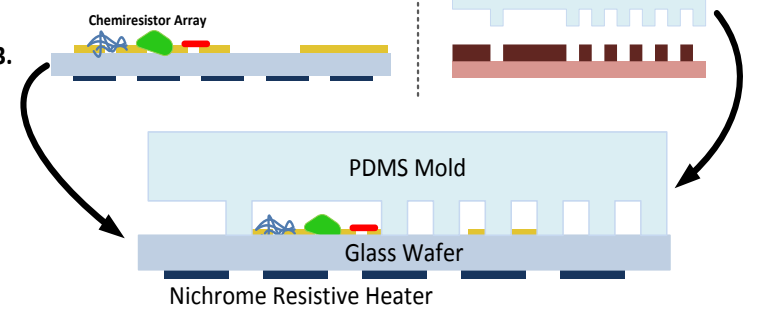

Figure 4 - Fabrication of micromolded gas chromatography device.

It should be noted that the fabrication method is very low cost. Almost every step can be performed with inexpensive benchtop equipment. The exception is the photolithography processes. However, even the photolithography is not especially 
technically challenging with critical dimensions greater than $20 \mu \mathrm{m}$.

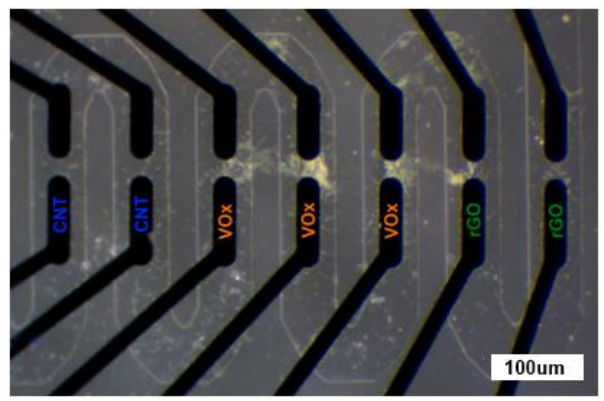

Figure 5 - Micrograph of chemiresistor array

\section{Gas analysis}

High purity (>99.999\%) nitrogen gas was used as the carrier gas regulated to 40psi. An analytical standard calibration set of aliphatic compounds (Sigma-Aldrich part number 4-8244) was used to verify the function of the device. This set contains unfunctionalized carbon chains ranging in length from 12 to 17 carbon atoms dissolved dichloromethane.

After waiting for the temperature of the device to stabilize (approximately 10 minutes), the gas was injected per the procedure outlined in the introduction. A simple circuit (shown in Figure 5) was developed to monitor the chemiresistor array. The circuit consists of three portions: a variable current source, an analog switch to select a single chemiresistor array element, and a voltage readout buffer. Continuous monitoring of an entire array is made possible by time multiplexing or scanning through the array. The circuit was designed to be compact to allow for future integration onto the gas chromatography device without significant size expansion. The readout circuitry connects to a laptop via a LabView data acquisition board (NI-6009) and software interface.
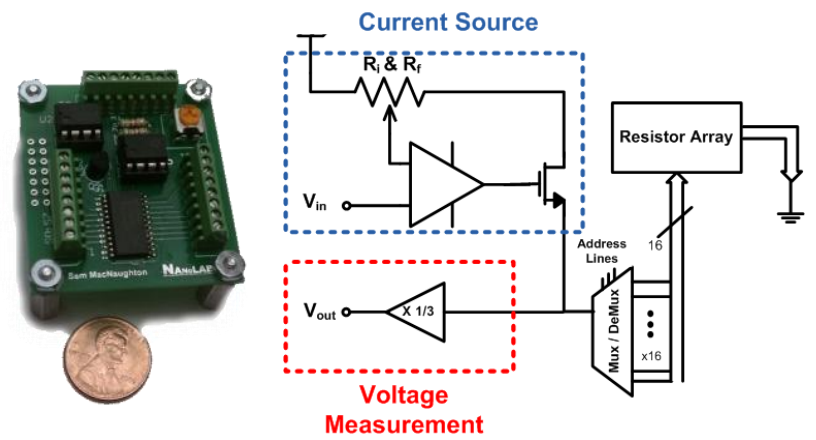

Figure 6-Image and schematic of monitoring electronics for the chemiresistor array detector.

The resulting chromatograph is shown in Figure 7, and a zoomed in portion of the rGO trace is shown in Figure 8 with the peaks identified. Each of the six peaks resultant from the six components of the analytics standard is clearly resolvable. A trace from a CNT assembly is omitted as they did not respond. It is hypothesized that the oxygen plasma treatment for bonding the glass wafer to the PDMS rendered the CNTs inert to the gas analytes.

As noted in the introduction, some limitations of chemiresistors are clearly evident. The magnitude of the responses decrease over time regardless of the analyte concentration. While this could be attributed to the peaks being widened or sampled at points other than their maximum, it is most likely due to the memory effect of the chemiresistors.
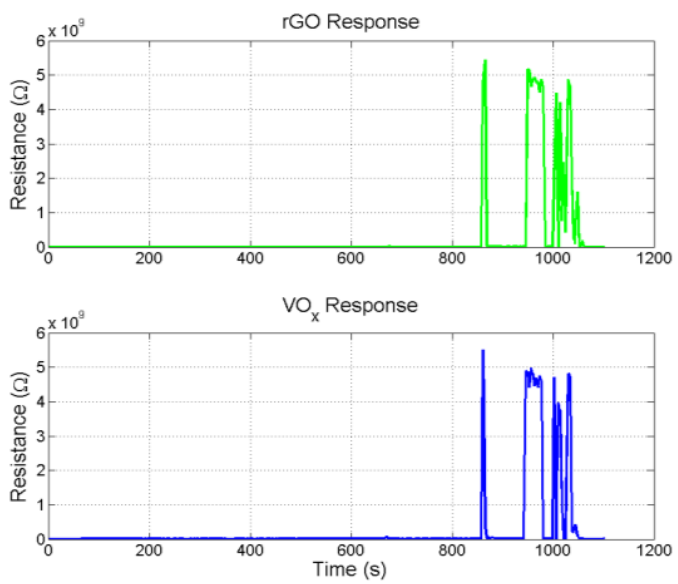

Figure 7-Chromatograph for aliphatic compound analytical standard. The sample was introduced at 650s, once the temperature and chemiresistor resistance had stabilized.

The typical gauge of the separation efficiency of is the theoretical number of plates. This is given by the following relation:

$$
N=5.54\left(\frac{t_{r}}{W_{0.5}}\right)^{2}
$$

$N$ is the number of plates, $t_{r}$ is the retention time, and $W_{0.5}$ is the half-width at full height of the peak. Calculating with respect to dodecane (which would give the worst case number) yields 4650 or 465 plates per meter. This value is comparable to other low power $\mu \mathrm{GC}$ devices found in the literature fabricated with the conventional etching and anodic bonding paradigm $[2,7,8]$.

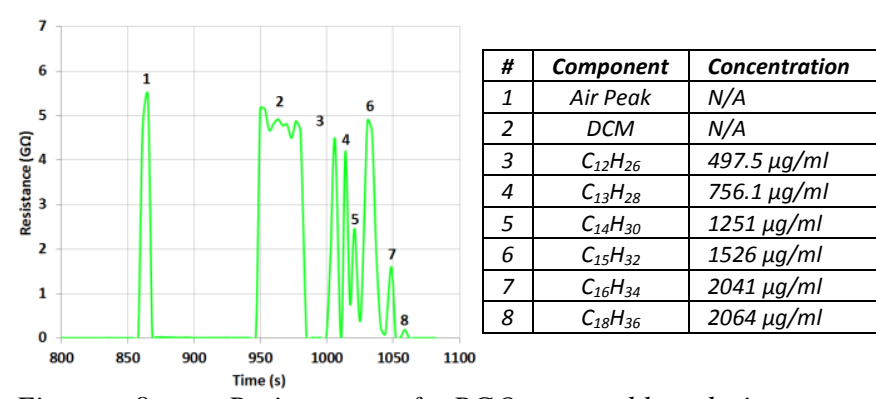

Figure 8 - Resistance of RGO assembly during gas chromatography run. It should be noted that resistances greater than $5 G \Omega$ could not be recorded due to hardware limitations.

\section{DISCUSSION}

We present the first micromolded PDMS column. The injection mechanism, heating element and a chemiresistor array detector were all integrated into a single device through a facile, inexpensive fabrication flow. This demonstrates the capability to integrate every necessary component into a small scale, low cost device for measurements in the field. The power consumption of less than three watts is in line with what is necessary for a portable device.

Furthermore, the addition of chemiresistor array as the 
detector adds a potential new dimension upon which to identify gas constituents based upon the characteristic response of a gas analyte across the elements of the array.

Further investigation is being pursued on many facets of this design. The integration of the passive valves in the PDMS channel and the electronics onto the device would eliminate all the external components used in the gas measurement. Using air as a carrier gas is also being investigated to eliminate the need for a tank of carrier gas, which is by far most bulky component of a gas chromatography measurement.

\section{REFERENCES}

[1] S. C. Terry, J. H. Jerman, and J. B. Angell, "A gas chromatographic air analyzer fabricated on a silicon wafer," Electron Devices, IEEE Transactions on, vol. 26, pp. 1880-1886, 1979.

[2] M. Agah, J. A. Potkay, G. Lambertus, R. Sacks, and K. D. Wise, "High-performance temperature-programmed microfabricated gas chromatography columns," Microelectromechanical Systems, Journal of, vol. 14, pp. 1039-1050, 2005.

[3] F. G. Bessoth, O. P. Naji, J. C. Eijkel, and A. Manz, "Towards an on-chip gas chromatograph: the development of a gas injector and a dc plasma emission detector," Journal of Analytical Atomic Spectrometry, vol. 17, pp. 794-799, 2002.
[4] S. MacNaughton, S. Sonkusale, S. Surwade, S. Ammu, and S. Manohar, "Electronic nose based on graphene, nanotube and nanowire chemiresistor arrays on silicon," in IEEE Sensors 2011, Hawaii, 2011, pp. 125-128.

[5] Y. W. Chang, J. S. Oh, S. H. Yoo, H. H. Choi, and K.-H Yoo, "Electrically refreshable carbon-nanotube-based gas sensors," Nanotechnology, vol. 18, p. 435504, 2007.

[6] C.-L. Chen, V. Agarwal, S. Sonkusale, and M. R. Dokmeci, "The heterogeneous integration of single-walled carbon nanotubes onto complementary metal oxide semiconductor circuitry for sensing applications," Nanotechnology, vol. 20, p. 225302, 2009.

[7] J. A. Potkay, G. R. Lambertus, R. D. Sacks, and K. D. Wise, "A low-power pressure-and temperature-programmable micro gas chromatography column," Microelectromechanical Systems, Journal of, vol. 16, pp. 1071-1079, 2007.

[8] H.-s. Noh, P. J. Hesketh, and G. C. Frye-Mason, "Parylene gas chromatographic column for rapid thermal cycling," Microelectromechanical Systems, Journal of, vol. 11, pp. 718-725, 2002.

\section{CONTACT}

*S. Sonkusale, tel: +1-617-627-5113; sameer@ece.tufs.edu 